\title{
Security wire mesh weaving process modelling with artificial neural network
}

\author{
Kridsada Wongwan ${ }^{1}$, Wimalin Laosiritaworn ${ }^{1}$ \\ ${ }^{1}$ Department of Industrial Engineering, Chiang Mai University, 239, Huay Kaew Road, Muang District, Chiang Mai, 50200, Thailand
}

\begin{abstract}
This paper investigates weaving process in the production of security woven wire mesh. Weaving is a critical process of the entire production as the quality of the final product depends very much on this process. High defect rate and low production yield is now a major concern in the production. There has been no prior study of the relationship among variables such as inspection data and machine setting on production yield. Conducting experiments to investigate this relationship is not reasonable in this case, as the product targeted at premium market and scrap cost is very high. In order to investigate the effect of these parameters, artificial neural network (ANN) was applied to model the process with data from the company databases. The type of ANN used in this research was the multi-layer neural network trained with back-propagation algorithm. The results suggested that ANN can effectively be used to predict weaving process production yield. The use of ANN proposed in this research is not limit to only weaving process, but can be applied to other manufacturing process.
\end{abstract}

\section{Introduction}

Woven wire mesh industry has expanded continuously globally. In Thailand, currently, there are more than 97 wire mesh manufacturers and distributors. Global steel consumption is forecasted to grow at an average of $1.6 \%$ per year to 1,814 million tons by 2020 , driven by the growth of emerging economies. Furthermore, US and South Korea's steel consumption is reported to grow by an average of $0.5 \%$ and $1.5 \%$ per year, respectively [1]. In order to deal with increasing demand in the expansion market, manufacturer need to improve their efficiency.

This research presents a case study from a large scale manufacturer of stainless steel wire security mesh manufacture located in northern Thailand. This high quality wire mesh is used in door and window assemble which targeted at premium market. The company produce over a million square meters of wire mesh per year. Security mesh (Figure 1) is not only very strong but unlike the traditional bars and grills, it is aesthetic as it is almost invisible, therefore unobstructed views. It also allows pest and UV protection, energy saving and can be customize to fit any type of doors and window.

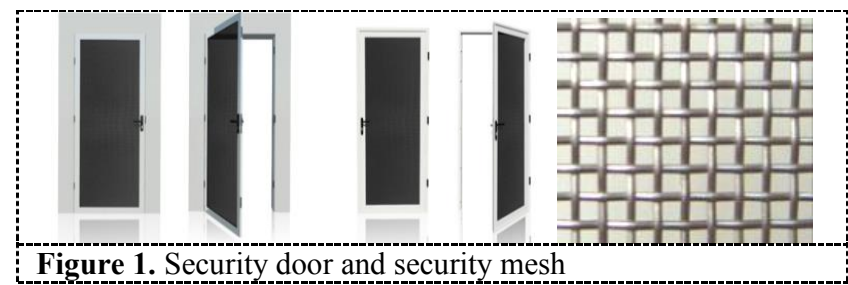

The production process of woven wire mesh consists of 8 main processes as shown in Figure 2. The production starts with material receiving process responsible by warehouse (WH) and quality assurance department (QA). In this process, raw material from suppliers is inspected for type, quantity, dimension, appearance defect. The next process is beaming which is responsible by weaving department $(\mathrm{BW})$. In this process the wire from pails or drums are rolled into flange of the bobbin. The number of wires in each flange are varied based on the width size of woven wire mesh requested by customer. Flange of bobbins then go to weaving processes responsible by $\mathrm{BW}$ department. In this process, the wires are set up into weaving machine with 2 directions, warp and weft wire direction, with different type of weaving pattern such as plain weave or twilled weave. The next process is cutting and inspection responsible by BW and QA department. In this process, the wire mesh rolls are unrolling and stretch into flat sheet, then they are cut and inspect of mesh defect by appearance. Inspection includes, for example, measuring of width and height, mesh count and aperture size. After inspection, the wire mesh sheets go to surface finishing process responsible by surface finishing department (SF) for pretreatment, powder coating and baking. This process is capable of coating on both of stainless and aluminum material. The next process is fabrication where wire mesh is cut and assemble for window or door product then pass to packing process and ready for shipping to customer. 


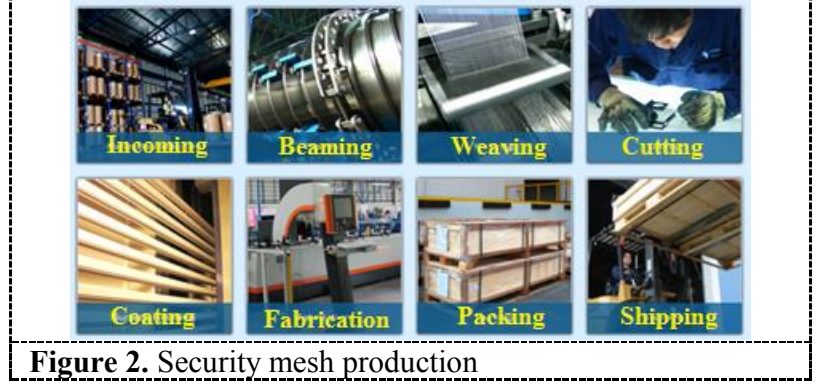

Defect is now a main problem in production. Figure 3 shows defect rate from 2012 to 2016 . It can be seen that the defect rate is steadily increased from $9.5 \%$ in 2012 to $13.7 \%$ in 2016 . The company target is to keep the rate lower than $5 \%$. Top defects are open mesh full defect (OF), hard warp defect (HP), open mesh defect (OM) and creeper defect (CP) as shown in Figure 4.
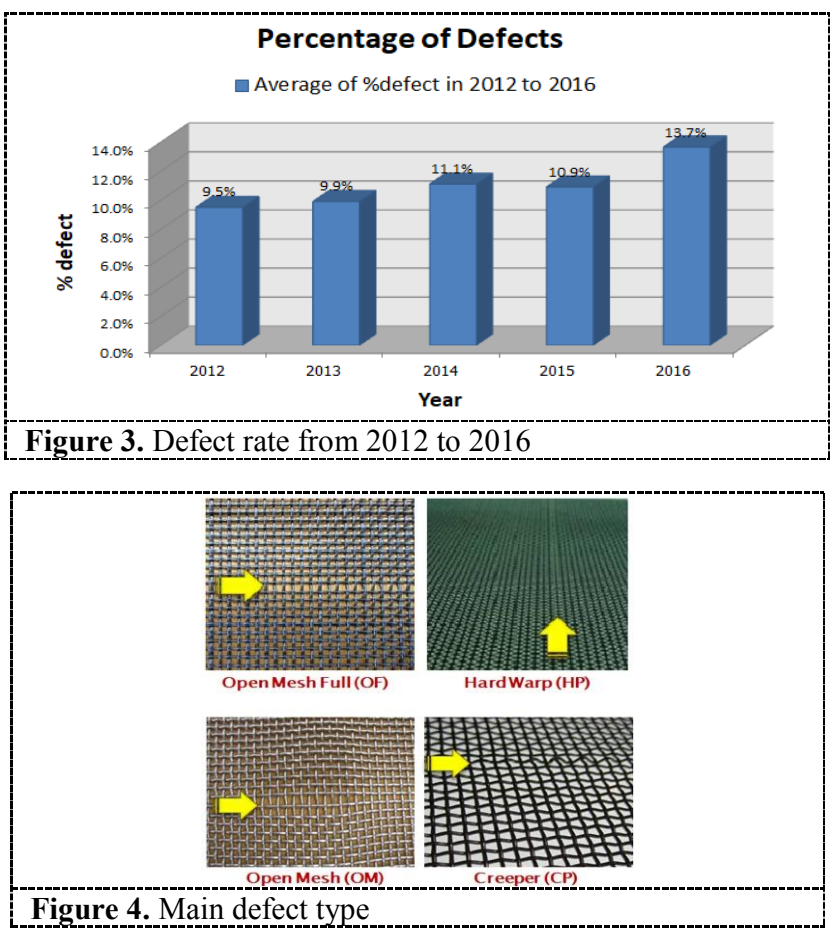

Weaving process is the critical process in the production. A number of factors including incoming inspection result, weaving machine and their setting could have affect on process yield. Currently, when quality problem occurs, weaving machine is adjusted by trial and error which take time and cost money as it might result in scrap. The relationship between these parameters and the process yield has not been studied before. If these relationships are well defined, better setting of the parameters can be achieved which ultimately leads to defect reduction. Experiments could be carried out with various parameters and process yield could be analyzed. However, conducting experiments involving stopping production and some experiment could result in defect. As, the scrap cost is of this product is very high, it will be very useful if a process model can be construed from production data available in the company's database.

Artificial neural network (ANN), one of the machine learning techniques, has been widely used in various function of manufacturing [2-4]. One of the possible applications of ANN is for process modeling in which ANN is trained to model relationship between process parameter setting and their corresponding quality characteristic. ANN has the capability to learn relationship complex relationship from training examples without task-specific programming. There have been number of reports of success case from various process, for example coil baking process [5], plasma etching [6], and superplastic forming process[7].

This paper describes an application of ANN to model weaving process parameter in order to predict process yield. Data used for training ANN were collected from the company databases. These data were used to train the network using 10 -fold cross validation method. After training, the model can be used to predict process yield. The rest of the paper is organized as follows; Background theory of ANN is covered in section 2. In section 3, the research methodology was explained and the experimental result is provided in section 4. Finally, section 5 is the conclusion of this research.

\section{Background theories}

\subsection{Introduction of artificial neural network}

ANN is by definition "an interconnected assembly of simple processing elements, units or nodes, whose functionality is loosely based on the animal neuron. The processing ability of the network is stored in the interunit connection strengths or weights, obtained by a process of adaptation to, or learning from, a set of training patterns" [8]. ANN allows computer to learn from example by creating a system that resemble animal nerve. Animal neuron is transformed in to artificial neuron which function shown in Figure 5.
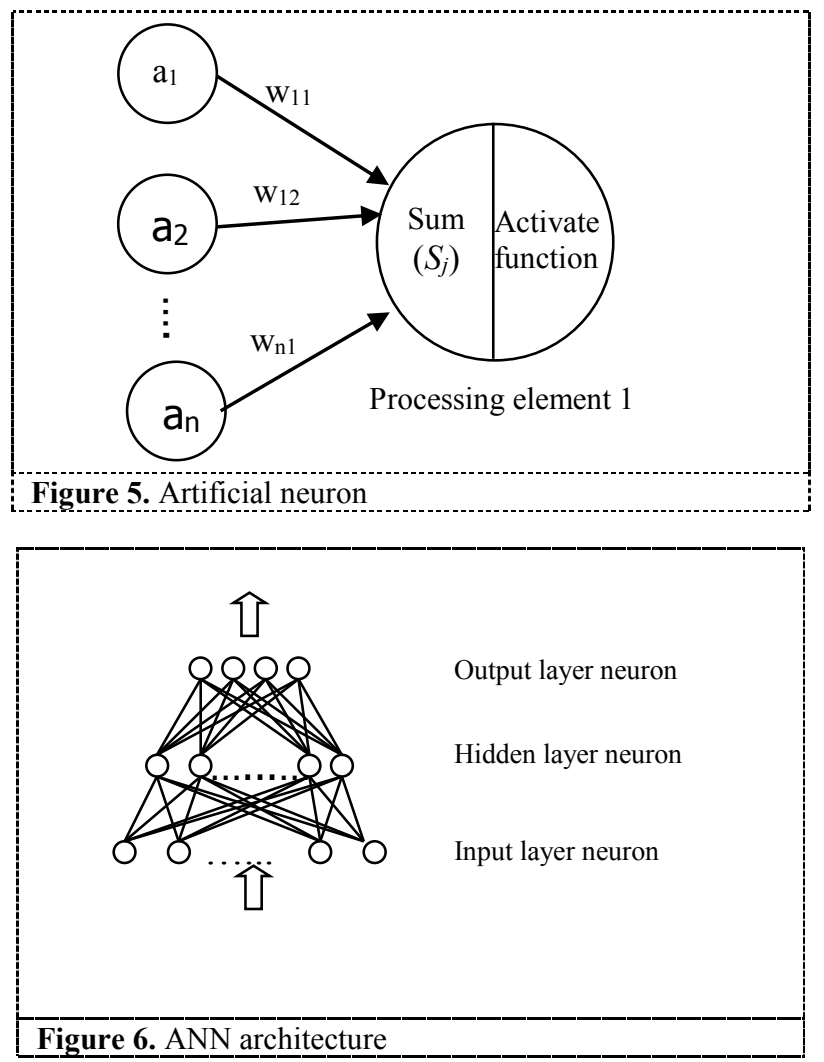
Many type of neural networks are available. A multilayer neural network trained with back- propagation algorithm is the most extensively adopted network [9]. In multi-layer neural network, neurons are organized in layer (Figure 6). Typical multi-layer neural network consists of 3 type of layers, input layer, hidden layer and output layer. Neurons in the input layer takes data from the outside of a neural network and sends it to hidden layer neurons. The output layer sends information back outside the network. Each neuron is connected to neuron in other layer as represent by straight line. The strength of the connections is representing by numbers called weight.

\subsection{ANN training algorithm}

Training process is a process in which weight between each neuron are altered to the value that minimise output error. Training start by setting initial weight into small random number. Then a set of examples of input-output pairs are passed through the model and the weights adjust in order to minimize the error between the answer from the network and the desired outputs. The weight adjustment procedure is controlled by the training algorithm. Once the error is minimal, the network is successfully trained. The trained network is able to predict output for unseen input. In this study, the BackPropagation algorithm. The algorithm can be summarized as follows [10].

Initially performs a 'forward pass', where the input is fed through the network to attain the output by calculate weighted sum $\left(S_{j}\right)$ for every neuron.

$$
S_{j}=\sum_{i} a_{i} w_{i j}
$$

where $a_{i}$ is the activation level of unit $i$, and $w_{i j}$ is the weight from unit $i$ to unit $j$. Then, the logistic transfer function, i.e. $g(x)=\frac{1}{1+e^{-x}}$ where $\mathrm{x}=S j$, were applied to the output. Then, $g\left(x=S_{j}\right)$ becomes the output of unit $j$, and the same procedure repeats for all neurons.

After that, the Back-Propagation performs a 'backward pass', where the error is calculated to update (adjust) the weight for each neuron for the output layer using the following equation.

$$
\delta_{j}=\left(t_{j}-a_{j}\right) g^{\prime}\left(S_{j}\right)
$$

For hidden layer equation 3 was used

$$
\delta_{j}=\left[\sum_{k} \delta_{k} w_{k j}\right] g^{\prime}\left(S_{j}\right)
$$

In these equations, $t_{j}$ is the target value for unit $j, a_{j}$ is the output value for unit $j, g^{\prime}(x)$ is the derivative of the logistic function $g$ and $S_{j}$ is weighted sum of inputs to $j$. Then, the weight adjustment is calculated as $\Delta w_{j i}=\eta \delta_{j} a_{i}$ where $\eta$ is the learning rate. These forward and backward processes repeat with new input vector until stopping criteria are met.

\section{Research methodology}

\subsection{Data collection from the company databases}

Data related to yield prediction is collected from 5 databases, as shown in Figure 7, which are chemical testing, mechanical testing, beaming process, weaving process and inspection process databases. Chemical testing database collect raw materials (wire) chemical composition testing such as carbon, molybdenum, nickel percentage. Mechanical testing database is related to mechanical properties testing of raw materials such as wire diameter size, tensile strength, offset yield and elongation. Beaming process database collect data regarding beaming process parameter such as beaming number, width size, number of rpm, tension type and tension level. Weaving process database records weaving parameters setting such as roll number, tension, weft density, tension, speed, timing, weft tensile and weft offset yield. Finally, inspection or quality control database collect quality inspection data for each roll of wire mesh.

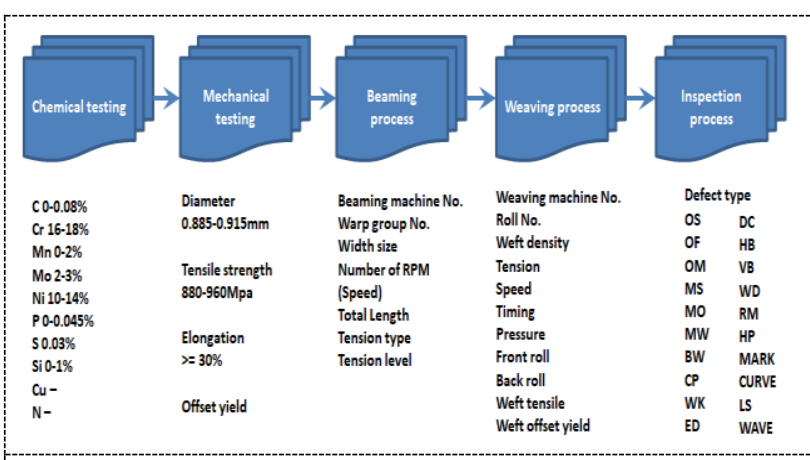

Figure 7. Databases relation to yield prediction

Among these data available, 9 parameters, shown in Table 1, which potentially has impact on production yield were selected as input for ANN. 816 records of data were collected from the company database to be used for ANN training. Table 1 also shows the type of parameter (input or output to $\mathrm{NN}$ ), unit, range and average value of the training data. The first 7 parameters are weaving process parameter obtained from weaving process databased. The first parameter is weaving machine number varied from 1 to 18 for 18 weaving machine in the shop floor. The second parameter is width size of the wire mesh. Wire mesh width size varied according to customer order but normally the width size is between $1.118 \mathrm{~m}$ to $2.018 \mathrm{~m}$ for $0.8 \mathrm{~mm} / 316$ product. The third parameter is weaving tension which is the tension force setting of weaving machine. Past records show that it was set between $7500 \mathrm{lb}$ to $8500 \mathrm{lb}$. The forth parameter is weaving speed which is usually set between 30 to $85 \mathrm{cycle} / \mathrm{min}$. The fifth parameter is timing which is the speed cycle of weaving roll rotation usually set between 328 to 350 . The sixth parameter is back roll level of weaving machine which is usually adjusted between -20 to 15 . The seventh parameter is front roll level of weaving machine which is usually adjusted between -20 to 10 . The next two parameters are related to mechanical testing of the incoming wire. The eighth parameter is tensile strength which has the value between $801 \mathrm{Mpa}$ to $964 \mathrm{Mpa}$. The last input parameter is the wire's yield point which is the offset yield or yield point of the which the value varied between $426 \mathrm{Mpa}$ to $946 \mathrm{Mpa}$. All these input parameters 
are used to predict the percentage of production yield which is calculated from the fraction of the square meters of the product that pass the quality control divided by the total square meters of the product manufactured.

Table 1. Parameters for NN modelling

\begin{tabular}{lccccc}
\hline Parameter name & Type & Unit & Min & Max & Average \\
\hline $\begin{array}{l}\text { Weaving machine } \\
\text { number }\end{array}$ & Input & n/a & & & \\
Wire mesh width size & Input & meter & 1.118 & 2.018 & 10.527 \\
Weaving tension & Input & Pound & 7500 & 8500 & 7818.260 \\
Weaving speed & Input & Cycle per minute & 30 & 85 & 59.185 \\
Timing & Input & n/a & 328 & 350 & 336.350 \\
Back roll level & Input & level & -20 & 15 & -3.027 \\
Front row level & Input & level & -20 & 10 & -6.483 \\
Wire tensile strength & Input & Megapascal & 801.0 & 964.0 & 911.472 \\
Wire yield strength & Input & Megapascal & 426.9 & 946.0 & 546.497 \\
Production yield & Output & Percent & 0.380 & 0.970 & 0.862 \\
\hline
\end{tabular}

\subsection{ANN architecture and parameter setting}

Input layer of ANN consist of 9 neurons for each input from Table 1 and output layer consist of 1 neuron for yield value. Important parameters for ANN are hidden neuron in hidden layer, learning rate and momentum. ANNs were trained by varying parameter according to Table 2. As hidden node in layer 1 has 6 levels (from 5 to 10 nodes), hidden node in layer 2 has 10 levels (from 0 to 9 nodes), learning rate and momentum has 4 and 5 levels respectively, $1200(6 \times 10 \times 4 \times 5)$ ANN were trained. The best ANN was selected based on root mean square error.

Table 2. Setting of ANN parameters

\begin{tabular}{lc}
\hline Parameter name & Level \\
\hline Hidden node in layer 1 & $5,6,7,8,9,10$ \\
Hidden node in layer 2 & $0,1,2,3,4,5,6,7,8,9$ \\
Learning rate & $0.2,0.3,0.4,0.5$ \\
Momentum & $0.1,0.2,0.3,0.4,0.5$ \\
\hline
\end{tabular}

\subsection{ANN training}

ANNs were implemented with RapidMiner software which is a leading software platform for implementing data preparation, machine learning and predictive model deployment. ANNs were trained with 10-fold cross validation method. In 10 -fold cross validation, 816 records of data used for training ANN was split into 10 subgroups. Data from 9 subgroups were used for training, leaving one subgroup out for testing. The process was repeated 10 times with different testing data from each subgroup. Then the average performance was calculated. This method provides a more accurate estimation of model performance.

\section{Results and discussion}

\subsection{RapidMiner process for ANN implementation}

In RapidMiner, task such as loading file or other calculation function were executed via "Operator". Each operator has specific input and output and perform specific task. By connecting line between operators, RapidMiner "process" is formed to do a series of task. RapidMiner process used in this research is shown in Figure 8.

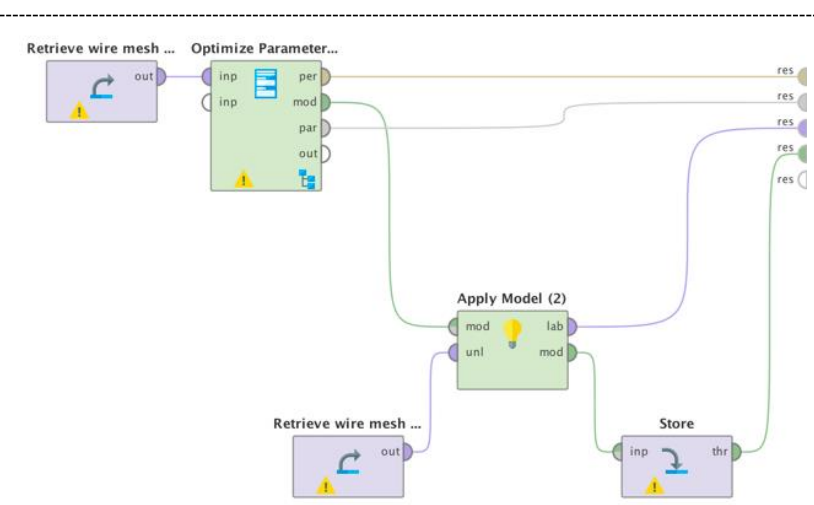

a) main process

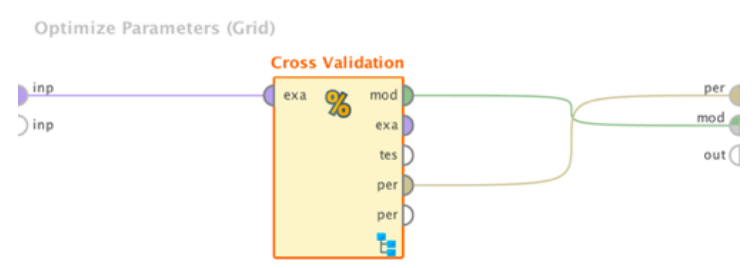

b) operator inside 'Optimize parameter' operator

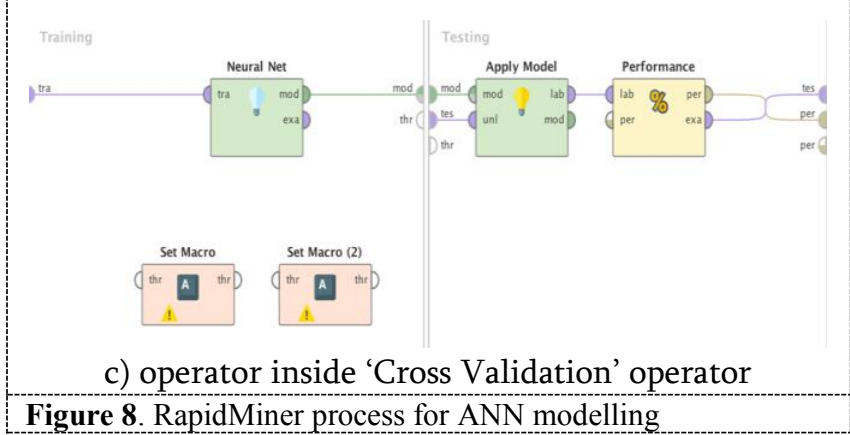

Figure 8 a) show RapidMiner's main process which start from loading wire mesh training file from repository. The file then passed through 'Optimize Parameter' operator which is a nested operator (more operator inside this operator). This operator allows user to search for the best parameter by conducting grid search with a set of user defined parameter level. In 
this work, parameters were set according to Table 2 . The best model obtained is send out from port 'mod' and is applied to predict new production data with 'Apply Model' operator. The optimized ANN model is stored back to repository for further analysis with operator 'Store'. In side 'Optimize Parameter' operator is a cross validation (Figure $8 \mathrm{~b}$ ) where 10-fold cross validation is applied for each condition setting in optimize parameter operator. Figure 8c) is a process inside 'cross validation' operator. In this step, ANN is constructed with 'Neural Net' operator with training data and tested the performance with testing data. 'Set Macro' operators allow hidden nodes to be varied during optimization process.

\subsection{Optimized ANN}

Figure 9 shows the optimized ANN architecture from the search. The best ANN results in RMSE of $0.0613 \pm 0.008$. This network has 9 neurons in the first hidden layer, 8 neurons in the second hidden layer and was trained with learning rate and momentum equal to 0.2. One extra neurons in each layer in Figure 5 are bias nodes that has constant input.

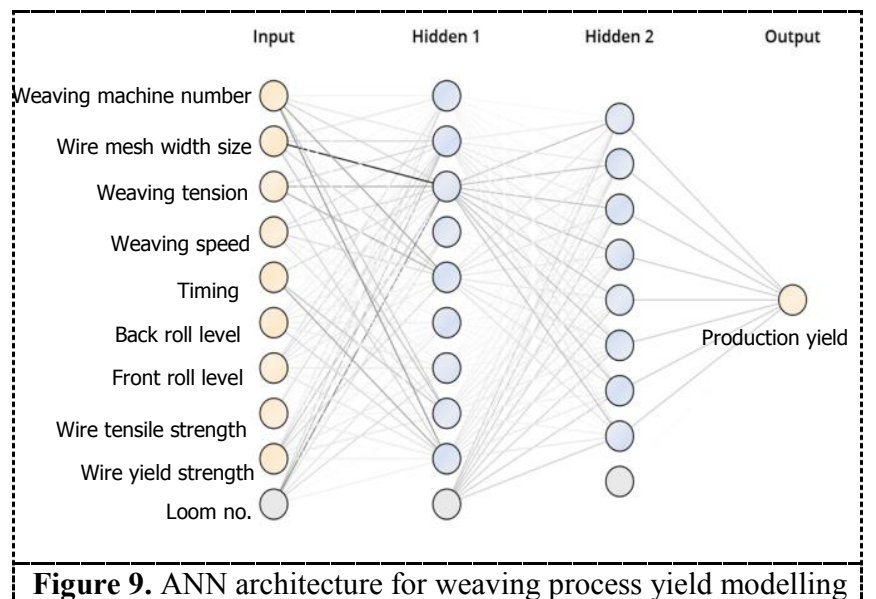

\subsection{ANN predication}

To test the accuracy of the model, 20 records of new data were collected. These data are unseen data which have never been used in the training and testing process before. The plot between ANN prediction and actual yield is shown in Figure 10.

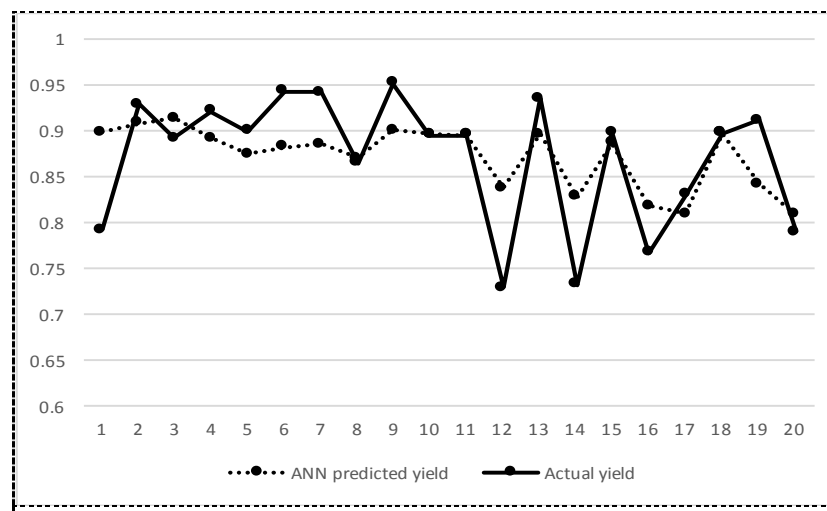

Figure 10. Plot between ANN predicted yield and actual yield

As can be seen from Figure 10 that ANN can reasonably pick up the relationship among variable and predict the yield. It can predict production lot that has the potential of low yield (such as lot number 12, 14, 16, 17, 18 and 20). However, in some lot especially lot number 12 and 14 even though it can predict that both lot will have low yield but the error of actual value could still be improved.

\section{Conclusion}

This paper presents an application of ANN to predict the production yield of weaving process for security wire mesh production. The research starts with collection data from the company's databased. Those data were combined together, resulting in the total of 816 records. They were used to train ANNs with 10fold cross validation method with varying ANN parameters. Among, 1,200 ANNs trained, the best ANN was the one with 9 neurons in the first hidden layer, 8 neurons in the second hidden layer and was trained with learning rate and momentum equal to 0.2 . The optimized ANN was used to predict new unseen data of 20 records. It was found that network was able to pick up the relationship among variable well and provide reasonable estimate of production yield. For further work, other techniques such as support vector machine or ensemble method could be used to improve the accuracy of the prediction even further.

\section{References}

1. The Federation of Thai Industries [internet]. 2015. Available from: http://ftiweb.off.fti.or.th/Backoffice/ ckfinder/userfiles/files/Steel\%20Situation $\% 20$ for $\% 2$ 0Mem_April\%202015(TH).pptx.

2. Köksal G, Batmaz İ, Testik MC. A review of data mining applications for quality improvement in manufacturing industry. Expert Systems with Applications. 2011;38(10):13448-67. 
3. Paliwal M, Kumar UA. Neural networks and statistical techniques: A review of applications. Expert Systems with Applications. 2009;36(1):2-17.

4. Krycha KA, Wagner U. Applications of artificial neural networks in management science: a survey. Journal of Retailing and Consumer Services. 1999;6(4):185-203.

5. Laosiritaworn WS, Coil Baking Process Modeling with Neural Network. IEEE International Conference on Industrial Engineering and Engineering Management; 2012; Hong Kong.

6. Rietman EA, Lory ER. Use of neural networks in modeling semiconductor manufacturing processes: an example for plasma etch modeling.
Semiconductor Manufacturing, IEEE Transactions on. 1993;6(4):343-7.

7. Sukthomya W, Tannock J. The training of neural networks to model manufacturing processes. Journal of Intelligent Manufacturing. 2005;16(1):13.

8. Gurney K. An Introduction to Neural Networks. London: UCL Press Limited; 1997.

9. Nascimento CAO, Giudici R, Guardani R. Neural network based approach for optimization of industrial chemical processes. Computers \& Chemical Engineering. 2000;24(9-10):2303-14.

10. Dayhoff JE. Neural Network Architectures: An Introduction. New York, USA: Van Nostrand Reinhold; 1990. 